\title{
ANALYTICAL FIELD CALCULATION OF HELICAL DIPOLE MAGNETS FOR RHIC
}

\author{
T. Tominaka, M. Okamura*, and T. Katayama** \\ The Institute of Physical and Chemical Research (RIKEN), Wako, Saitama, 351-01, Japan \\ *Brookhaven National Laboratory, Upton, NY 11973-5000, USA \\ **Center for Nuclear Study, School of Science, University of Tokyo, Tanashi, Tokyo, 188, Japan
}

\begin{abstract}
The analytical expression for the magnetic field of helical coils is presented. In particular, the multipole coefficients are introduced for helical dipole magnets, which are essential components of Siberian Snakes and spin rotators for polarized proton acceleration at RHIC. In addition, the comparison between analytical and numerical calculations is presented for simple helical dipole magnets.
\end{abstract}

\section{INTRODUCTION}

Using the expression of multipole expansion for a single helical current conductor[1], the magnetic field of helical dipole coil with an infinite length is derived as the summation of four(4) helical line currents with dipole symmetry, deriving the helical multipole coefficients. $[2,3]$ The helical multipole coefficients are defined so that the non-twist helical multipole coefficients are equal to the 2-dimensional multipole coefficients. As a result, the dependence of helical multipole coefficients upon the twist parameter is derived. With the comparison between the analytical and numerical calculations, it is confirmed that the helical multipole coefficients derived from the analytically calculated field are consistent with those calculated numerically.

\section{ANALYTICAL EXPRESSION FOR THE MAGNETIC FIELD OF HELICAL DIPOLES}

Using the expression of the multipole expansion for a single helical current conductor, the magnetic field inside the helical coils with an infinite length can be derived as the summation of four(4) helical line currents with dipole symmetry as follows, [1,2,3]

$$
\left\{\begin{array}{c}
\mathrm{B}_{\mathrm{r}}(\mathrm{r}, \theta, \mathrm{z})=\mathrm{B}_{\mathrm{ref}}(\mathrm{k}) \mathrm{r}_{0} \sum_{\mathrm{n}=1}^{\infty} \mathrm{n} !\left[\frac{2}{\mathrm{n} \mathrm{k} \mathrm{r} \mathrm{r}_{0}}\right]^{\mathrm{n}} \mathrm{k} \mathrm{I}_{\mathrm{n}}(\mathrm{n} \mathrm{k} \mathrm{r}) \times \\
\left\{-\mathrm{a}_{\mathrm{n}}(\mathrm{k}) \cos \left(\mathrm{n}(\theta-\mathrm{k} \mathrm{z}) \mid+\mathrm{b}_{\mathrm{n}}(\mathrm{k}) \sin (\mathrm{n}(\theta-\mathrm{k} \mathrm{z}))\right\}\right. \\
\mathrm{B}_{\theta}(\mathrm{r}, \theta, \mathrm{z})=\mathrm{B}_{\mathrm{ref}}(\mathrm{k}) \mathrm{r}_{0} \sum_{\mathrm{n}=1}^{\infty} \mathrm{n} !\left[\frac{2}{\mathrm{n} \mathrm{k} \mathrm{r} \mathrm{r}_{0}}\right]^{\mathrm{n}} \frac{\mathrm{I}_{\mathrm{n}}(\mathrm{n} \mathrm{k} \mathrm{r})}{\mathrm{r}} \times \\
\left\{\mathrm{a}_{\mathrm{n}}(\mathrm{k}) \sin (\mathrm{n}(\theta-\mathrm{k} \mathrm{z}))+\mathrm{b}_{\mathrm{n}}(\mathrm{k}) \cos (\mathrm{n}(\theta-\mathrm{k} \mathrm{z}))\right\} \\
\mathrm{B}_{\mathrm{z}}(\mathrm{r}, \theta, \mathrm{z})=\mathrm{B}_{\mathrm{ref}}(\mathrm{k}) \mathrm{r}_{0} \sum_{\mathrm{n}=1}^{\infty}(-\mathrm{k}) \mathrm{n} !\left[\frac{2}{\mathrm{n} \mathrm{k} \mathrm{r}_{0}}\right]^{\mathrm{n}} \mathrm{I}_{\mathrm{n}}(\mathrm{n} \mathrm{k} \mathrm{r}) \times \\
\left\{\mathrm{a}_{\mathrm{n}}(\mathrm{k}) \sin (\mathrm{n}(\theta-\mathrm{k} \mathrm{z}))+\mathrm{b}_{\mathrm{n}}(\mathrm{k}) \cos (\mathrm{n}(\theta-\mathrm{k} \mathrm{z}))\right\},
\end{array}\right.
$$

Therefore, the normal and skew multipoles due to many helical line currents with current $\mathrm{I}_{\mathbf{i}}$, radius $\mathrm{a}_{\mathbf{i}}$, angle $\varphi_{\mathrm{i}}$ are,

$$
\begin{aligned}
& \left(\mathrm{B}_{\mathrm{n}}(\mathrm{k})=\mathrm{B}_{\mathrm{ref}}(\mathrm{k}) \mathrm{b}_{\mathrm{n}}(\mathrm{k})=-\frac{\mu_{0}}{\pi} \frac{1}{2^{\mathrm{n}}(\mathrm{n}-1) ! \mathrm{r}_{0}}\left(\mathrm{n} \mathrm{k} \mathrm{r}_{0}\right)^{\mathrm{n}} \times\right. \\
& \sum_{i} I_{i}\left(k a_{i} K_{n-1}\left(n k a_{i}\right)+K_{n}\left(n k a_{i}\right)\right) \cos n \varphi_{i} \\
& \mathrm{~A}_{\mathrm{n}}(\mathrm{k})=\mathrm{B}_{\mathrm{ref}}(\mathrm{k}) \mathrm{a}_{\mathrm{n}}(\mathrm{k})=-\frac{\mu_{0}}{\pi} \frac{1}{2^{\mathrm{n}}(\mathrm{n}-1) ! \mathrm{r}_{0}}\left(\mathrm{n} \mathrm{k} \mathrm{r}_{0}\right)^{\mathrm{n}} \times \\
& \sum_{i} I_{i}\left(k a_{i} K_{n-1}\left(n k a_{i}\right)+K_{n}\left(n k a_{i}\right)\right) \sin n \varphi_{i}
\end{aligned}
$$

where $k=2 \pi / L$, and $I_{n}(n k r)$ and $K_{n}(n k r)$ are the modified Bessel functions of the first and second kind of order $n$, respectively. With the definition of $\mathrm{b}_{1}(\mathrm{k})=1$ (=constant), naturally $\mathrm{B}_{\mathrm{ref}}(\mathrm{k})=\mathrm{B}_{1}(\mathrm{k})$. Then, the asymptotic forms for the reference field $\mathrm{B}_{\mathrm{ref}}(\mathrm{k})$ and for these helical multipole coefficients $a_{n}(k)$ and $b_{n}(k)$ are given in the limit $\mathrm{k} \rightarrow 0$ (or $\mathrm{L} \rightarrow \infty$ ) by,

$$
\left\{\begin{aligned}
\lim _{k \rightarrow 0}\left[B_{\text {ref }}(k)\right] & =B_{\text {ref }} \\
\lim _{k \rightarrow 0}\left[a_{n}(k)\right] & =a_{n} \\
\lim _{k \rightarrow 0}\left[b_{n}(k)\right] & =b_{n}
\end{aligned}\right.
$$

\section{COMPARISON BETWEEN ANALYTICAL AND NUMERICAL CALCULATIONS}

\subsection{Calculation for four helical line currents}

Following numerical values are assumed in the calculation for four helical line currents with dipole symmetry.

Radius of helical line current: $\mathrm{a}=50 \mathrm{~mm}$, Angle of helical line current: $\varphi= \pm \pi / 6$, or $\pm 5 \pi / 6$,

Current: $\mathrm{I}=-1 \times 10^{5} \mathrm{~A}$,

Pitch length: $\mathrm{L}=2 \mathrm{~m}$,

$\mathrm{k}=2 \pi / \mathrm{L}=1 /(\mathrm{a} \tan \alpha)=\pi$, 


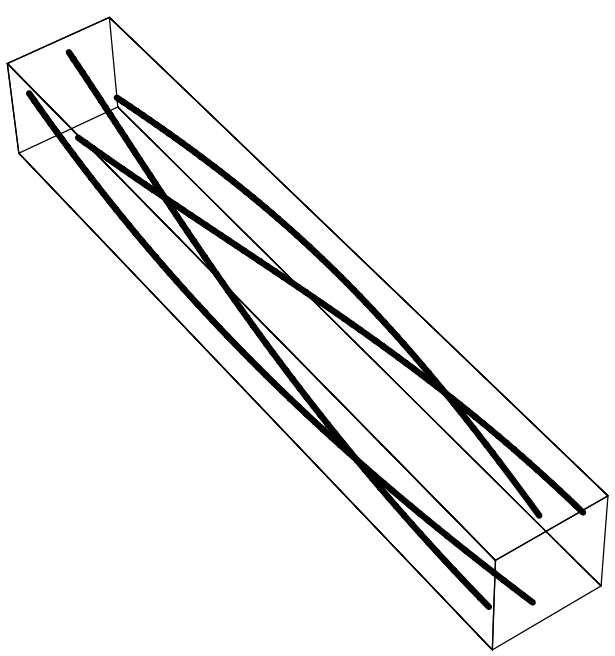

Fig. 1. 3D view of a four currents' helical dipole.

Table 1. Normal multipole coefficients for a infinitely long four line currents' helical dipole.

\begin{tabular}{llll}
$\mathrm{n}$ & Pole & bn-theta & bn $(\mathrm{k})$ \\
\hline (Bref) & & 1.411 & 1.411 \\
1 & dipole & 0.9998 & 1. \\
3 & sextupole & $-2.85910^{-10}$ & 0 \\
5 & decapole & -0.1239 & -0.1243 \\
7 & 14 -pole & -0.04386 & -0.04419 \\
9 & 18 -pole & -1.72110 & 0
\end{tabular}

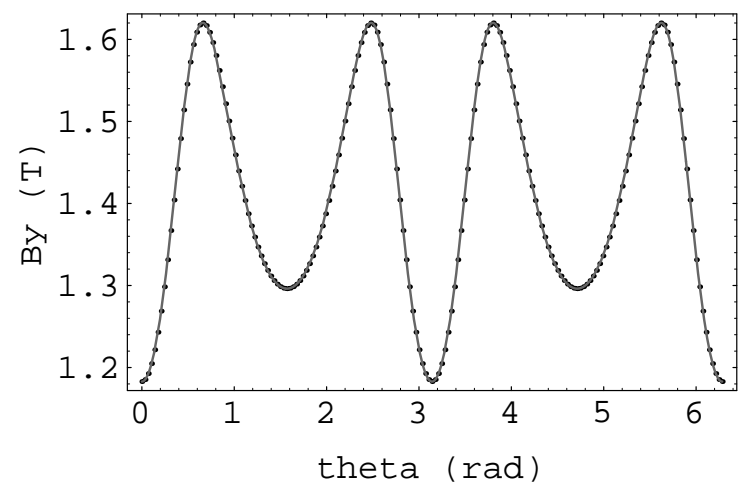

Fig. 3. Distribution $B_{y}$ on the circle of $r=30 \mathrm{~mm}$, with the comparison between the numerical (black dots) and analytical (gray line) calculations.

Pitch of the winding: $\alpha=\tan ^{-1}(1 / 0.05 \pi)$,

Reference radius for multipole: $\mathrm{r}_{0}=30 \mathrm{~mm}$,

as shown in Fig.1. Both of the numerical and analytical calculations for the helical dipoles with the infinite length, are made, using Mathematica [4]. Both calculated helical multipole coefficients are listed in Table 1. The

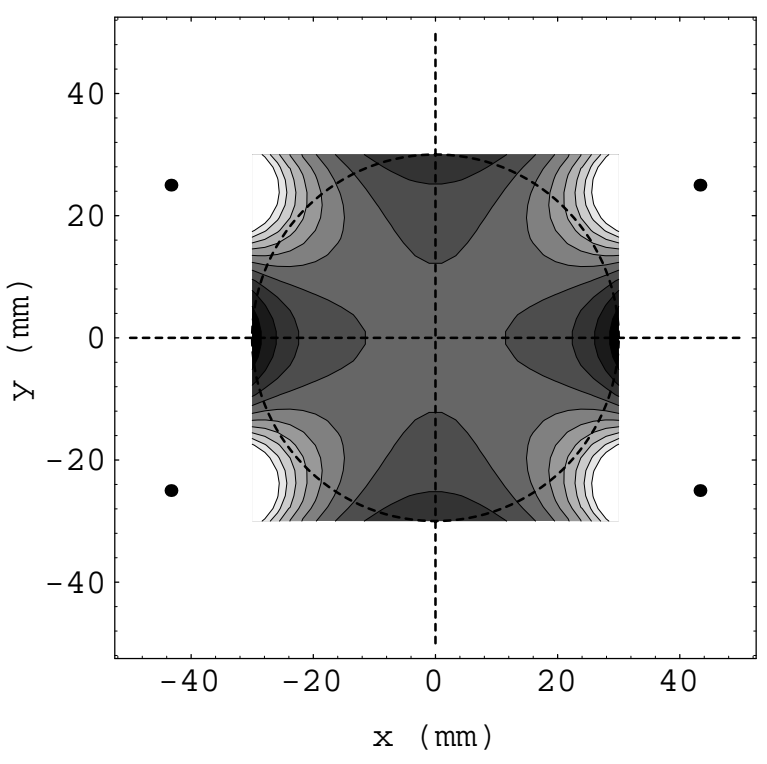

Fig. 2. Contour plot of dipole field $\mathrm{B}_{\mathrm{y}}$.

bn-theta and $b_{n}(k)$ correspond to the normal multiple coefficients derived from the angular component of numerically calculated field $\mathrm{B}_{\theta}$ and analytically calculated helical coefficients, respectively. The analytically calculated contour plot of the dipole field $\mathrm{B}_{\mathrm{y}}(\mathrm{r}, \theta, \mathrm{z}=0)$ is shown in Fig.2. Both analytical (gray line) and numerical (black dots) calculations for the dipole field $\mathrm{B}_{\mathrm{y}}(\mathrm{r}=30 \mathrm{~mm}$, $\theta, \mathrm{z}=0$ ) are also shown in Fig.3. As a result, it is confirmed that the agreement between the analytical and numerical calculations is quite good in the interior region of helical coils. The twist dependence of the dipole field $\mathrm{B}_{\text {ref }}$ is also shown in Fig.4.

\subsection{Calculation for four helical current shells}

Similarly, the magnetic fields are calculated for a four helical current shells with dipole symmetry of current density $\pm \mathrm{j}_{\mathrm{Z}}$, radii $\mathrm{a}_{1}, \mathrm{a}_{2}$, limiting angles $\pm \varphi_{1}, \pm \varphi_{2}$, with,

Inner radius of helical line current: $\mathrm{a}_{1}=50 \mathrm{~mm}$, Outer radius of helical line current: a2 $=60 \mathrm{~mm}$, Inner angle of helical line current: $\varphi_{1}=0$, or $\pi$, Outer angle of helical line current: $\varphi_{2}=\pi / 3$, or $2 \pi / 3$, Coil length: $\mathrm{L}=2 \mathrm{~m}$,

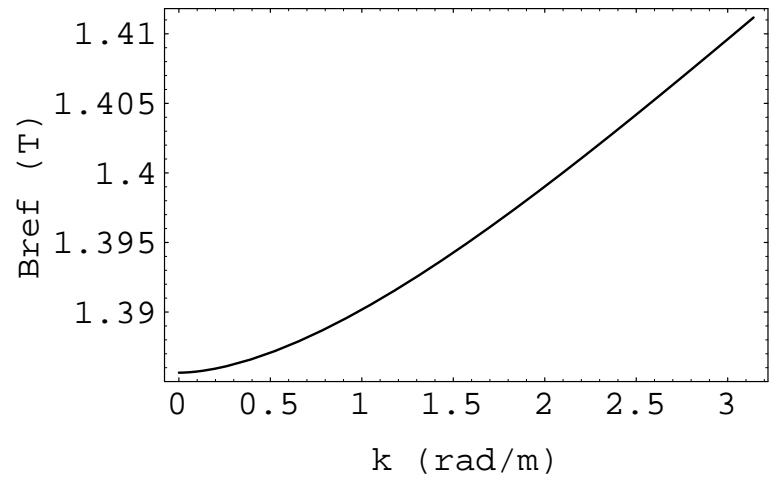

Fig. 4. Twist dependence of the dipole field $\mathrm{B}_{\text {ref. }}$ 


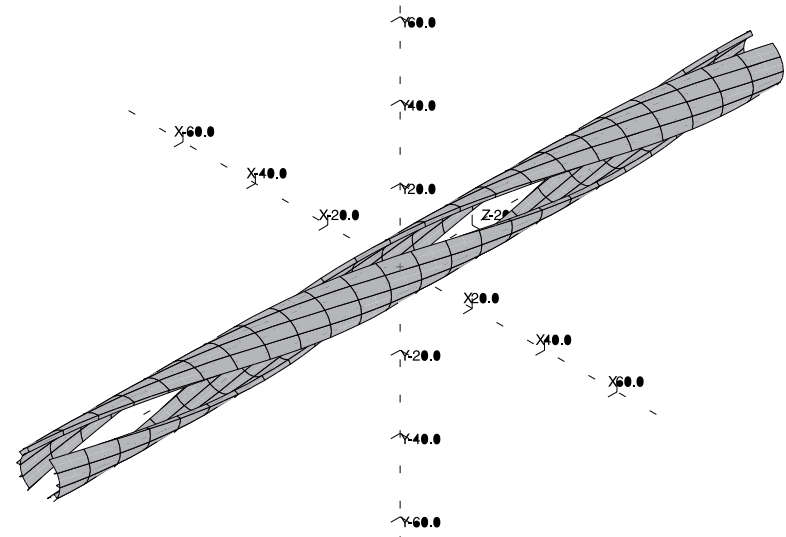

Fig.5. 3D view of a four helical current shells' dipole.

Table 2. Normal multipole coefficients for a $2 \mathrm{~m}$ long, 4 helical current shells' dipole.

\begin{tabular}{llll}
$\mathrm{n}$ & Pole & bn-theta & bn $(\mathrm{k})$ \\
\hline (Bref) & & 2.463 & 2.456 \\
1 & dipole & 1. & 1. \\
3 & sextupole & $-1.00110^{-6}$ & $1.36910^{-17}$ \\
5 & decapole & -0.01727 & -0.01734 \\
7 & 14 -pole & $0.003718^{-6}$ & 0.003743 \\
9 & 18 -pole & $-3.24610^{-19}$ & $3.77510^{-19}$
\end{tabular}

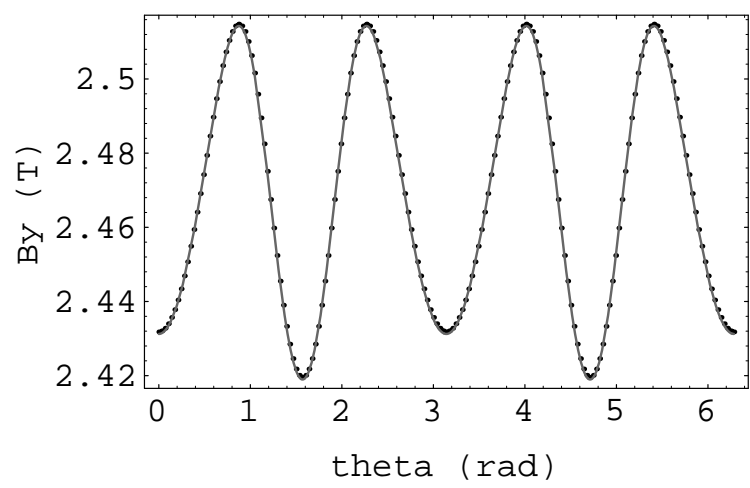

Fig. 6. Distribution of By on the circle of $r=30 \mathrm{~mm}$.

Current: $\mathrm{I}=-2 \times 10^{5} \mathrm{~A}$,

Current density: $\mathrm{j}_{\mathrm{z}}=347 \mathrm{~A} / \mathrm{mm}^{2}$

$\mathrm{k}=2 \pi / \mathrm{L}=1 /(\mathrm{a} \tan \alpha)=\pi$,

Pitch of the winding: $\alpha=\tan ^{-1}(1 / 0.05 \pi)$,

Reference radius for multipole: $\mathrm{r}_{0}=30 \mathrm{~mm}$,

as shown in Fig.5. The numerical calculation for the helical coil with the finite length of one period is made, using the code 'OPERA-3d'. [5] Both analytical (gray line) and numerical (black dots) calculations for the dipole field $\mathrm{B}_{\mathrm{y}}(\mathrm{r}=30 \mathrm{~mm}, \theta, \mathrm{z}=0)$ is shown in Fig.6. The 3D

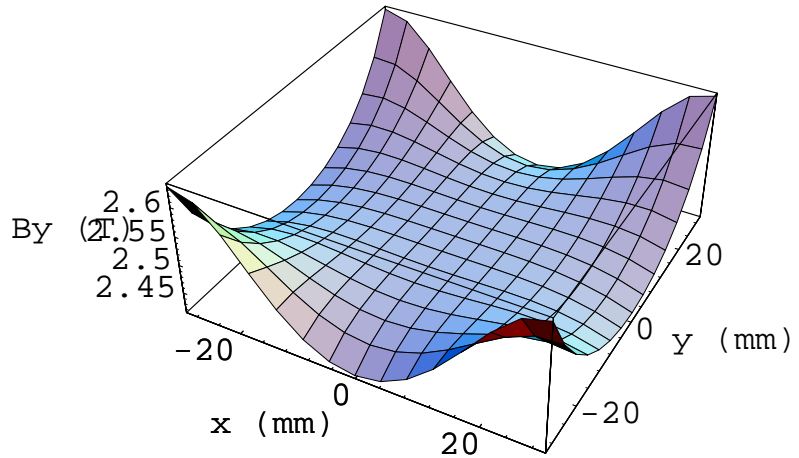

Fig. 7. 3D plot of dipole field By.

plot of the analytically calculated dipole field $B_{y}(r, \theta$, $\mathrm{z}=0$ ) is shown in Fig.7. Both of the analytically and numerically calculated helical multipole coefficients are also listed in Table 2. In addition, this analytical method was applied for two helical magnet of different type. $[6,7,8]$

\section{CONCLUSION}

As an extension of single helical current conductor, the magnetic fields of helical dipole magnet are derived and helical multipole coefficients are presented. In addition, it was confirmed that this analytical calculation is consistent with the numerical calculation by the $3 \mathrm{D}$ magnetic field numerical code, OPERA-3d/TOSCA

\section{ACKNOWLEDGMENTS}

The authors are indebted for helpful discussions and comments to the RIKEN RHIC spin accelerator group.

\section{REFERENCES}

[1] T. Tominaka et al., "Multipole Expansion for a Single Helical Current Conductor", in this proceedings.

[2] T. Tominaka, "Multipole Expansion for a Single Helical Current Conductor", AGS/RHIC/SN No.49, December 18, (1996).

[3] T. Tominaka, "Magnetic Field Calculation of Helical Dipole Coils", AGS/RHIC/SN No.24, April 29, (1996).

[4] Stephen Wolfram, "Mathematica, A system for Doing Mathematics by Computer", Addison-Wesley Publishing Company, Inc., (1991).

[5] Vector Fields Limited, Oxford, England.

[6] T. Tominaka, "Analytical Field Calculation of the Slotted Helical Dipole", AGS/RHIC/SN No.47, November 12, (1996).

[7] T. Tominaka, "Analytical Field Calculation of the Direct Wind Helical Dipole", AGS/RHIC/SN No.48, December 18, (1996).

T. Tominaka, " Relation between Field Homogeneity and Multipole Coefficients for a Helical Dipole", AGS/RHIC/SN No.54, April 25, (1997). 